\title{
Sunspots, Correlation and Competition
}

\author{
Herakles Polemarchakis*and Indrajit Ray ${ }^{\dagger}$
}

September 2003

\begin{abstract}
Correlated equilibria in strategic market games played, simultaneously, by "overlapping generations" of players correspond to sunspot equilibria in the associated, competitive economy. The lower the degree of competition, the larger the range of parameters that allow for effective correlation or endogenous stochastic fluctuations.

Keywords: Sunspot equilibria; Correlated equilibria; Market games.

JEL Classification Numbers: C72, D50, D82, E30.
\end{abstract}

${ }^{*}$ Department of Economics, Brown University, Providence, RI 02912, USA. E-mail: herakles_polemarchakis@brown.edu; Fax: +1.401.863.1970.

${ }^{\dagger}$ Author for Correspondences. Department of Economics, University of Birmingham, Edgbaston, Birmingham B15 2TT, UK. E-mail: i.ray@bham.ac.uk; Fax: +44.121.414.7377. 


\section{Introduction}

Extrinsic uncertainty has been extensively discussed in competitive equilibrium theory and macroeconomic theory since the pioneering contributions of Azariadis (1981) and Cass and Shell (1983). Sunspot equilibria represent endogenous stochastic fluctuations in market economies, as cycles, in Benhabib and Day (1982) or Grandmont (1985), represent endogenous deterministic fluctuations.

Correlated equilibria for non-cooperative games, introduced by Aumann $(1974,1987)$ bear an evident similarity with sunspot equilibria for competitive markets and, no wonder, several have explored the possible connection between these two notions ${ }^{1}$.

Our purpose here is to study

1. the connection between correlated equilibrium and sunspot equilibrium in the simplest possible framework: an economy of overlapping generations in which individuals are strategic ${ }^{2}$;

\footnotetext{
${ }^{1}$ Maskin and Tirole (1987) considered a two-period, two-good, two-type model of an imperfectly competitive market and proved that extrinsic uncertainty can indeed affect the equilibrium, but only if there is imperfect correlation - neither perfect correlation nor complete independence. Aumann, Peck and Shell (199?) constructed an example of an imperfectly correlated or sunspot equilibrium of a market game with two types of individuals. Forges (1991) considered extensive, rather than strategic form correlated equilibria (Forges $(1986,1988)$ ), and proved that they coincide with sunspot equilibria for a market game with complete security markets (Postlewaite and Schmeidler, (1978), Peck, Shell and Spear (1992)). Peck and Shell (1991) defined sunspot-Nash equilibria in market games with state-contingent security markets and proved that correlated equilibrium allocations are also sunspot-Nash equilibrium allocations with vanishing trades in securities. Peck (1994) compared correlated and sunspot equilibria using the models of Azariadis (1981) and Cass and Shell (1983). Forges and Peck (1995) proved that the sunspot equilibria of a standard economy of overlapping generations and the correlated equilibria of the multi-stage market game that mimics it are equivalent. Davila (1999) considered correlated equilibria of a two-period, two-good, two-type economy as in Maskin and Tirole (1987) and sunspot equilibria of a market game played by the associated economy of overlapping generations and provided conditions under which the two coincide.

${ }^{2}$ Overlapping generations models with strategic agents have already been studied. Goenka, Kelly and Spear (1998) studied complex and chaotic equilibrium dynamics as in Grandmont (1985). Jacobsen (2000) considered imperfectly competitive cycles, while Heinemann (1997)
} 
2. the extent to which the degree of competition affects the possibility of endogenous fluctuations.

The strategic behavior of individuals or the imperfectly competitive structure of markets we model as a market game, following Shapley (1976) or Shapley and Shubik (1977). Importantly, the game is played simultaneously by a countable infinity of individuals or players, whose preferences and endowments parallel the structure of an economy of overlapping generations of Samuelson (1958) ${ }^{3}$; this eliminates considerations of dynamic or extensive form strategic choice and of refinements, such as, subgame perfection. Forges (1991), in the concluding remarks of her article, suggested the usefulness of this simple model for the study of extrinsic uncertainty: "It could be worthwhile to investigate this model or more sophisticated versions of it by studying the extensive form correlated equilibria ... and by replicating the players to get results on the limit economy." Forges and Peck (1995) mentioned this game as well, and they suggested the construction of the correlated equilibrium that we use here.

Azariadis (1981) used the simple model of overlapping generations and a simple, two-state, stationary Markov process to show that the resulting equilibria may be subject to extrinsic uncertainty; and he gave necessary and sufficient conditions for the existence of a (non-trivial) two-state, stationary sunspot equilibrium. Here we construct a correlation device for this normal form game and then study the normal form, symmetric correlated equilibrium.

Nash equilibria in a strategic market game converge to competitive equilibria of the associated economy - Dubey and Shubik (1978); this is the case in our set up as well. Convergence fails in Cordella and Datta (2002), which is, still, a conundrum.

discussed rationalizable sunspots in such a setup. Cordella and Datta (2002) characterized and compared competitive (Walras) and strategic (Cournot-Nash) stationary equilibria.

${ }^{3}$ It is now well understood that the fundamental features of competitive equilibria in economies of overlapping generations derive from the double infinity of individuals and commodities and not from the temporal structure - Geanakoplos (1987) and Geanakoplos and Polemarchakis (1991). 
We show that

1. there is a natural parallel between stationary Markov correlated equilibria in a market game and stationary Markov equilibria with sunspots in the associated competitive economy, and any differences vanishes as the economy gets large;

2. the lower is the degree of competition, the larger is the range of parameters that allow for effective correlation.

Further work should consider general settings.

\section{The Set up}

Types of individuals are $t=0,1, \ldots$, and individuals of each type are $i=$ $1, \ldots, n$; an individual is, thus, $(i, t)$ or simply $t$, when only the type of the individual matters.

Commodities are $\tau=1, \ldots$

Individuals of type $t \neq 0$, are endowed with and derive utility from the consumption of commodities $\tau=t$ and $\tau=(t+1)$.

The consumption of an individual is a bundle, $\left(x^{t}, y^{t}\right)$, of commodities $\tau=t$ and $\tau=(t+1)$, respectively.

The economy is stationary: an individual derives utility from consumption according to the (intertemporal) von Neumann - Morgenstern utility function

$$
w(x, y)=u(x)+v(y), \quad(x, y) \geq 0,
$$

and his endowment of commodities $\tau=t$ and $\tau=(t+1)$ is

$$
\omega=(e, f) \geq 0 .
$$

Individuals of type $t=0$, are endowed with and derive utility from the consumption of commodity $\tau=1$.

Such an economy in which there are exactly $n(\geq 2)$ individuals of each type, is denoted by $E_{n}$. 


\subsection{A strategic market game}

Agents are (strategic) players in the game and can influence the prices of the commodities by their buy and sell orders.

The strategy set of an individual of type $t \neq 0$ is

$$
\mathcal{S}^{i, t}=\left\{q_{t}^{i}: 0 \leq q_{t}^{i} \leq e\right\}
$$

with the interpretation that $q_{t}^{i}$ is the quantity of commodity $\tau=t$ that the individual offers in exchange for commodity $\tau=t+1$.

Individuals of type $t=0$ make no strategic choices.

A profile of strategies $\vec{q}=\left(q_{t}^{i}\right)$ determines the aggregate supply and, as a consequence, the price of every commodity, $\tau$, respectively, according to

$$
Q_{\tau}=\sum_{i} q_{\tau}^{i}, \quad \text { and } \quad p_{\tau}\left(Q_{\tau}\right)=\frac{1}{Q_{\tau}}
$$

The allocation of commodities to an individual of type $t \neq 0$ associated with a profile of strategies is

$$
x^{i, t}=e-q_{t}^{i}, \quad y^{i, t}=f+q_{t}^{i} \frac{p_{t}}{p_{t+1}},
$$

and his utility or payoff is

$$
w^{i, t}=u\left(x^{i, t}\right)+v\left(y^{i, t}\right)=u\left(e-q_{t}^{i}\right)+v\left(f+q_{t}^{i} \frac{p_{t}}{p_{t+1}}\right) .
$$

This is the formulation of Shapley (1976) and Shapley and Shubik (1977).

The aggregate consumption of individuals of type $t=0$ is $Q_{1}$.

\subsection{The associated competitive economy}

Prices of commodities are

$$
p=\left(p_{1}, \ldots, p_{\tau}, \ldots\right) \geq 0 .
$$

The budget constraint of an individual of type $t \neq 0$ is

$$
x^{i, t}=e-q_{t}^{i}, \quad y^{i, t}=f+q_{t}^{i} \frac{p_{t}}{p_{t+1}} .
$$


The individual demands or supplies commodities in order to maximize his utility subject to the budget constraint.

Individuals of type $t=0$ consume $Q_{1}$, the aggregate supply of the commodity.

The interpretation and the analogy with the model of overlapping generations are evident: commodities are exchanged for fiat money, a medium of exchange, in fixed supply normalized to 1 ; exchange of commodities for balances occurs sequentially according to $\tau$; the supply of balances complements the endowment of individuals of type $t=0$.

\subsection{Equilibria: correlation and sunspots}

A state of the world is $\vec{s}=\left(s_{t} ; t \neq 0\right)$, with $s_{t} \in\{a, b\}$. The information partition of individuals $t \neq 0$ is

$$
\mathcal{P}^{t}=\left\{P_{a}^{t}=\left\{\vec{s}: s_{t}=a\right\}, P_{b}^{t}=\left\{\vec{s}: s_{t}=b\right\}\right\}
$$

individuals $t=0$ are uninformed, which is immaterial, since they make no strategic decisions.

Probability distributions over states of the world are generated by the stationary Markov transition probabilities

$$
\left(\begin{array}{cc}
\pi(a \mid a) & \pi(a \mid b) \\
\pi(b \mid a) & \pi(b \mid b)
\end{array}\right)=\left(\begin{array}{cc}
\pi(a) & 1-\pi(a) \\
1-\pi(b) & \pi(b)
\end{array}\right) .
$$

With perfect serial correlation, $\pi(a \mid a)=\pi(b \mid b)=1$ or $\pi(a)=\pi(b)=1$.

With serial independence, $\pi(a \mid a)=\pi(a \mid b)$ and $\pi(b \mid b)=\pi(b \mid a)$ or $\pi(a)+$ $\pi(b)=1$.

This describes the correlation device.

A strategy of an individual, $t \neq 0$, is a pair $\left\{q_{t}(a), q_{t}(b)\right\}$ : the quantity supplied by the individual as a function of the information he receives.

A symmetric, stationary, Markov, correlated equilibrium for the strategic market game is described by transition probabilities and strategies, $\{\pi(a), \pi(b)$, 
$q(a), q(b)\}$, such that

$$
q(s)=\arg \max _{q} \mathrm{E}_{s^{\prime} \mid s} u(e-q)+v\left(f+q \frac{p(q+(n-1) q(s))}{p\left(s^{\prime}\right)}\right), \quad s, s^{\prime} \in\{a, b\},
$$

where, the stationary structure allows us to denote $s_{t}$ and $s_{t+1}$, by $s$ and $s^{\prime}$, respectively.

This is a symmetric, stationary, Markov Nash equilibrium of the game extended by the correlation device, as in Aumann (1974, 1987).

For the associated economy, a stationary, Markov, competitive equilibrium with sunspots is described by transition probabilities, prices of commodities and quantities supplied, $\{\pi(a), \pi(b), p(a), p(b), q(a), q(b)\}$, such that

$$
q(s)=\arg \max _{q} \mathrm{E}_{s^{\prime} \mid s} u(e-q)+v\left(f+q \frac{p(s)}{p\left(s^{\prime}\right)}\right), \quad s, s^{\prime} \in\{a, b\} .
$$

This is the formulation of Azariadis (1981).

As $n \rightarrow \infty$, the optimal responses of the agents in the correlated equilibrium of the strategic market game converge to those in the competitive equilibria with sunspots in the associated economy.

\section{Results}

We restrict attention to interior values: $0<q(a), q(b)<e$, as standard restrictions on preferences and endowments allow; also, to non-degenerate probability distributions: $0<\pi(a), \pi(b)<1$.

Lemma 1 A correlated equilibrium for the strategic market game, $\{\pi(a), \pi(b)$, $q(a), q(b)\}$, is a solution to the system of equations

$$
\begin{aligned}
& u^{\prime}(e-q(a))=\frac{n-1}{n}\left(\pi(a) v^{\prime}(f+q(a))+(1-\pi(a)) v^{\prime}(f+q(b)) \frac{q(b)}{q(a)}\right), \\
& u^{\prime}(e-q(b))=\frac{n-1}{n}\left(\pi(b) v^{\prime}(f+q(b))+(1-\pi(b)) v^{\prime}(f+q(a)) \frac{q(a)}{q(b)}\right) .
\end{aligned}
$$

A sunspot equilibrium for the associated competitive economy, $\{\pi(a), \pi(b)$, 
$p(a), p(b), q(a), q(b)\}$, is a solution to the system of equations

$$
\begin{gathered}
u^{\prime}(e-q(a))=\pi(a) v^{\prime}(f+q(a))+(1-\pi(a)) v^{\prime}(f+q(b)) \frac{q(b)}{q(a)}, \\
u^{\prime}\left(e-q(b)=\pi(b) v^{\prime}(f+q(b))+(1-\pi(b)) v^{\prime}(f+q(a)) \frac{q(a)}{q(b)} .\right.
\end{gathered}
$$

Proof. For the strategic market game, the result follows from the definition of a correlated equilibrium and the necessary and sufficient first order conditions for strategies mutual optimal responses.

For the associated competitive economy, the result follows from the definition of a equilibrium and the necessary and sufficient first order conditions for utility maximization subject to the budget constraint.

As the number of individuals in each type or generation, $n$, increases, correlated equilibria of the strategic market game coincide with sunspot equilibria of the associated economy. This is an application of the argument in Dubey and Shubik (1978), without reference to no-trade equilibria that arise naturally in market games irrespective of the size of the market.

Corollary 1 At a correlated equilibrium,

$$
\begin{aligned}
& \pi(a)=\frac{\frac{n}{n-1} u^{\prime}(e-q(a)) q(a)-v^{\prime}(f+q(b)) q(b)}{v^{\prime}(f+q(a)) q(a)-v^{\prime}(f+q(b)) q(b)}, \\
& \pi(b)=\frac{\frac{n}{n-1} u^{\prime}(e-q(b)) q(b)-v^{\prime}(f+q(a)) q(a)}{v^{\prime}(f+q(b)) q(b)-v^{\prime}(f+q(a)) q(a)} .
\end{aligned}
$$

At the corresponding sunspot equilibrium,

$$
\begin{aligned}
& \pi(a)=\frac{u^{\prime}(e-q(a)) q(a)-v^{\prime}(f+q(b)) q(b)}{v^{\prime}(f+q(a)) q(a)-v^{\prime}(f+q(b)) q(b)}, \\
& \pi(b)=\frac{u^{\prime}(e-q(b)) q(b)-v^{\prime}(f+q(a)) q(a)}{v^{\prime}(f+q(b)) q(b)-v^{\prime}(f+q(a)) q(a)} .
\end{aligned}
$$

Following Cass and Shell (1985), "extrinsic uncertainty is effective" or sunspots do matter, at a competitive equilibrium, if $q(a) \neq q(b)$; extrinsic uncertainty is effective if it is effective at some competitive equilibrium. Similarly, we shall say that correlation is effective, at a correlated equilibrium, if $q(a) \neq q(b)$; correlation is effective if it is effective at some correlated equilibrium. 
A correlated equilibrium where $q(a)=q(b)=q_{n}^{*}$ and, as a consequence, correlation is ineffective, exists: it obtains as the solution to the equation

$$
u^{\prime}(e-q)=\frac{n-1}{n} v^{\prime}(f+q)
$$

and it is the symmetric, stationary, interior Nash equilibrium in pure strategies of the normal form market game in discussion.

Similarly, a competitive equilibrium where $q(a)=q(b)=q^{*}$ and, as a consequence, sunspots are ineffective, exists: it obtains as the solution to the equation

$$
u^{\prime}(e-q)=v^{\prime}(f+q)
$$

and it is the stationary equilibrium of the associated competitive economy.

The existence of correlated equilibria with effective correlation depends on the curvature of the cardinal utility indices of individuals; equivalently, the slope of the supply of savings with respect to the rate of interest or the relative risk aversion of individuals over (net) second period consumption.

If at $q^{*}$, the (Nash) equilibrium, $v^{\prime \prime}\left(f+q^{*}\right) q^{*}+v^{\prime}\left(f+q^{*}\right)<0(>0)$, then, savings is an decreasing (increasing) function of the interest factor, $p_{t} / p_{t+1}$, and relative risk aversion over (net) second period consumption is higher (lower) than 1.

Simple characterizations obtain if the sign of the expression $v^{\prime \prime}(f+q) q+$ $v^{\prime}(f+q)$ does not vary with $q>0$; the argument is as in Azariadis (1981).

\section{Lemma 2 If}

$$
v^{\prime \prime}(f+q) q+v^{\prime}(f+q)>0
$$

then correlation is ineffective.

Proof. By the hypothesis, the function $v^{\prime}(f+q) q$ is strictly monotonically increasing, as is, by concavity, the function $u^{\prime}(e-q) q$.

We argue by contradiction:

Without loss of generality, $q(a)<q(b)$, and, as a consequence, $v^{\prime}(f+$ $q(a)) q(a)<v^{\prime}(f+q(b)) q(b)$. Since $\pi(a)<1$, from the expressions in Corollary $1,(n /(n-1)) u^{\prime}(e-q(a))>v^{\prime}(f+q(a))$, and, as a consequence, $q(a)>q_{n}^{*}$. 
Since $\pi(b)<1$, similarly, $q(b)<q_{n}^{*}$. It follows that $q(b)<q(a)$, a contradiction.

Lemma 3 With

$$
v^{\prime \prime}(f+q) q+v^{\prime}(f+q)<0,
$$

at a correlated equilibrium with $\pi(a)+\pi(b) \geq 1$, correlation is ineffective.

Proof. By the hypothesis, the function $v^{\prime}(f+q) q$ is strictly monotonically decreasing, while, from concavity, the function $u^{\prime}(e-q) q$ is monotonically increasing.

We suppose that $\pi(a)+\pi(b) \geq 1$ and argue by contradiction:

Without loss of generality, $q(a)<q(b)$, and, as a consequence, $v^{\prime}(f+$ $q(a)) q(a)>v^{\prime}(f+q(b)) q(b)$.

With $\pi(a) \geq(1-\pi(b))$, it follows from the expressions for $\pi(a)$ and $\pi(b)$ in Corollary 1, that $u^{\prime}(e-q(a)) \geq u^{\prime}(e-q(b))$, and, therefore, $q(a) \geq q(b)$, a contradiction.

Importantly, if the conditions necessary for effective correlation are satisfied, $\pi(a)+\pi(b)<1$, which excludes signals that are serially independent.

Lemma 4 With

$$
v^{\prime \prime}(f+q) q+v^{\prime}(f+q)<0
$$

correlation is effective only if

$$
-\frac{d}{d q} v^{\prime}(f+\hat{q}) \hat{q}>\frac{n}{n-1} \frac{d}{d q} u^{\prime}(e-\hat{q}) \hat{q}
$$

or, equivalently,

$$
-\left(v^{\prime \prime}(f+\hat{q}) \hat{q}+v^{\prime}(f+\hat{q})\right)>-\frac{n}{n-1} u^{\prime \prime}(f-\hat{q})+u^{\prime}(f-\hat{q}),
$$

at some $\hat{q}>0$.

Proof. Without loss of generality, $q(a)<q(b)$, and, as a consequence, $v^{\prime}(f+q(a)) q(a)>v^{\prime}(f+q(b)) q(b)$. 
Then, $0<\pi(a), \pi(b)<1$ if and only if

$$
\begin{aligned}
& v^{\prime}(f+q(b)) q(b)<\frac{n}{n-1} u^{\prime}(e-q(a)) q(a)<v^{\prime}(f+q(a)) q(a), \\
& v^{\prime}(f+q(b)) q(b)<\frac{n}{n-1} u^{\prime}(e-q(b)) q(b)<v^{\prime}(f+q(a)) q(a)
\end{aligned}
$$

or, equivalently,

$$
\begin{aligned}
& v^{\prime}(f+q(b)) q(b)<\frac{n}{n-1} u^{\prime}(e-q(a)) q(a), \\
& \frac{n}{n-1} u^{\prime}(e-q(b)) q(b)<v^{\prime}(f+q(a)) q(a) .
\end{aligned}
$$

These inequalities can be satisfied simultaneously only if, somewhere in their domain, the function $v^{\prime}(f+q) q$ decreases faster than the function $\frac{n}{n-1} u^{\prime}(e-q) q$ increases.

\section{Theorem 1 With}

$$
v^{\prime \prime}(f+q) q+v^{\prime}(f+q)<0
$$

if

$$
v^{\prime}(f+\underline{q}) \underline{q}>\frac{n}{n-1} u^{\prime}(e-\bar{q}) \bar{q},
$$

for some

$$
0<\underline{q}<\bar{q},
$$

and

$$
-\frac{d}{d q} v^{\prime}(f+\underline{q}) \underline{q}>\frac{n}{n-1} \frac{d}{d q} u^{\prime}(e-\underline{q}) \underline{q}
$$

or, equivalently,

$$
-\left(v^{\prime \prime}(f+\underline{q}) \underline{q}+v^{\prime}(f+\underline{q})\right)>-\frac{n}{n-1} u^{\prime \prime}(f-\underline{q})+u^{\prime}(f-\underline{q}),
$$

then correlation is effective.

Proof. It suffices to set $q(a)=\underline{q}$ and $q(b)=\bar{q}$.

Corollary 2 If correlation is effective at equilibrium with some $0<\underline{q}<\bar{q}$ satisfying the hypothesis in Theorem 1 , for some $\bar{n}$, then correlation is effective at equilibrium for all economies with $n \geq \bar{n}$. 
Proof. As $n$ increases, $((n-1) / n)$ increases or, equivalently, $(n /(n-1))$ decreases to 1 . If $\underline{q}<\bar{q}$ satisfies the hypothesis in the Theorem 1 for $\bar{n}$, then it does so for all $n \geq \bar{n}$.

From the theorem, it is clear that the degree of competition, $((n-1) / n)$, enhances the possibility of effective correlation: an increase in $n$, may allow for effective correlation.

The following parametric example illustrates the point.

Example 1 Take

$$
w(x, y)=u(x)+v(y)=-x^{-k}-x^{-2 k}, \quad 1 \leq k \leq 8,
$$

and

$$
\omega=(e, f)=(10,2) .
$$

For $k=1$ or $k=2, q(\alpha)=1$ and $q(\beta)=2$ is an equilibrium for any value of $n$.

For $k=3, q(\alpha)=1$ and $q(\beta)=2$ is an equilibrium only for $n>2$.

\section{Theorem 2 With}

$$
v^{\prime \prime}(f+q) q+v^{\prime}(f+q)<0
$$

if

$$
-\frac{d}{d q} v^{\prime}\left(f+q_{n}^{*}\right) q_{n}^{*}>\frac{n}{n-1} \frac{d}{d q} u^{\prime}\left(e-q_{n}^{*}\right) q_{n}^{*}
$$

or, equivalently,

$$
-\left(v^{\prime \prime}\left(f+q_{n}^{*}\right) q_{n}^{*}+v^{\prime}\left(f+q_{n}^{*}\right)\right)>-\frac{n}{n-1} u^{\prime \prime}\left(f-q_{n}^{*}\right) q_{n}^{*}+u^{\prime}\left(f-q_{n}^{*}\right),
$$

then correlation is effective.

Corollary 3 If $q_{n}^{*}$ satisfies the hypothesis in Theorem 2 in an economy $E_{n}$, and thereby correlation is effective at equilibrium, then correlation is effective at equilibrium for all economies $E_{n^{\prime}}, n^{\prime}>n$.

The degree of competition, $((n-1) / n)$, acts as a discount factor for second period consumption, and, as such, it affects the possibility of effective correlation. 
Since the cardinal utility indices for first and second period consumption need not coincide, there is no meaning to the numeric value of a discount factor.

More importantly, the effect of a discount factor or of the degree of competition is secondary, since it does not affect directly the curvature of the utility index.

The following parametric example illustrates these points.

Example 2 Take

$$
w(x, y)=u(x)+v(y)=x-\delta \frac{1}{\alpha} y^{\alpha}, \quad \alpha<1, \delta>0
$$

and

$$
\omega=(e, f)=(1,0)
$$

At the Nash equilibrium,

$$
q_{n}^{*}=\left(\frac{n-1}{n} \delta\right)^{\frac{1}{1-\alpha}}
$$

and

$$
\begin{gathered}
-\left(v^{\prime \prime}\left(f+q_{n}^{*}\right) q_{n}^{*}+v^{\prime}\left(f+q_{n}^{*}\right)\right)>-\frac{n}{n-1} u^{\prime \prime}\left(f-q_{n}^{*}\right) q_{n}^{*}+u^{\prime}\left(f-q_{n}^{*}\right) \\
\Leftrightarrow \\
\alpha<-1,
\end{gathered}
$$

independently of the degree of competition or the discount factor. 


\section{REFERENCES}

1. Aumann, R. J. (1974), "Subjectivity and correlation in randomized strategies," Journal of Mathematical Economics, 1, 67-96.

2. Aumann, R. J. (1987), "Correlated equilibrium as an expression of bayesian rationality," Econometrica, 55, 1-18.

3. Aumann, R. J., J. Peck and K. Shell (199?), "Asymmetric information and sunspot equilibria : a family of simple examples," in W. Kuhn (ed.), Incomplete Information and Bounded Rationality, Springer Verlag, 000000

4. Azariadis, C. (1981), "Self-fulfilling prophecies," Journal of Economic Theory, 25, 380-396.

5. Benhabib, J. and R. Day (1982), "A characterization of erratic dynamics in the overlapping generations model," Journal of Economic Dynamics and Control, 4, 37-55.

6. Cass, D. and K. Shell (1983), "Do sunspots matter?," Journal of Political Economy, 91, 193-227.

7. Cordella, T. and M. Datta (2002), "Intertemporal cournot and walras equilibria: an illustration," International Economic Review, 43, 137-153.

8. Davila, J. (1999), "On the connection between correlated equilibria and sunspot equilibria," in A. Alkan, C. D. Aliprantis, and N. C. Yannelis (eds.), Current Trends in Economics: Theory and Applications, Springer Verlag, 141-156.

9. Dubey, P. and M. Shubik, (1978), "The non-cooperative equilibria of a closed trading economy with market supply and bidding strategies," Journal of Economic Theory, 17, 1-20.

10. Forges, F. (1986), "An approach to communication equilibrium," Econometrica, 54, 1375-1385. 
11. Forges, F. (1988), "Can sunspots replace a mediator?," Journal of Mathematical Economics, 17, 347-368.

12. Forges, F. (1991), "Sunspot equilibrium as a game-theoretical solution concept," W. A. Barnett, B. Cornet, C. d'Aspremont, J. J.-Gabszewicz and A. Mas-Colell (eds.), Equilibrium Theory and Applications: Proceedings of the Sixth International Symposium in Economic Theory and Econometrics, Cambridge University Press, 135-159.

13. Forges, F. and J. Peck (1995), "Correlated equilibrium and sunspot equilibrium," Economic Theory, 5, 33-50.

14. Geanakoplos, J. D. (1987), "The overlapping generations model of general equilibrium, " in J. Eatwell, M. Milgate and P. Newman (Eds.) The new Palgrave: a dictionary of economics, Macmillan, 767-779.

15. Geanakoplos, J. D., and H. M. Polemarchakis (1991), "Overlapping generations," in W. Hildenbrand and H. Sonnenschein (Eds.) Handbook of Mathematical Economics, Vol. IV, North Holland, 1899-1948.

16. Goenka, A., D. Kelly and S. E. Spear (1998), "Endogenous strategic business cycles," Journal of Economic Theory, 81, 97-125.

17. Grandmont, J.-M. (1985), "On endogenous competitive business cycles," Econometrica, 53, 995-1045.

18. Heinemann, F. (1997), "Rationalizable expectations and sunspot equilibria in an overlapping-generations economy, Journal of Economics, 65, 257-277.

19. Jacobsen, H. J. (2000), "Endogenous, imperfectly competitive business cycles," European Economic Review, 44, 305-336.

20. Maskin, E. and J. Tirole (1987), "Correlated equilibria and sunspots," Journal of Economic Theory, 43, 364-373. 
21. Peck, J. (1994), "Notes on correlated equilibrium and sunspot equilibrium," in J.-F. Mertens and S. Sorin (eds.), Game Theoretic Methods in General Equilibrium Analysis, Kluwer, 249-260.

22. Peck, J. and K. Shell (1991), "Market uncertainty: correlated and sunspot equilibria in imperfectly competitive economies," Review of Economic Studies, 58, 1011-1029.

23. Peck, J., K. Shell, K. and S. E. Spear (1992), "The market game - existence and structure of equilibrium," Journal of Mathematical Economics, 21, 271-299.

24. Postlewaite, A. and D. Schmeidler (1978), "Approximate efficiency of nonwalrasian Nash equilibria," Econometrica, 46, 127-136.

25. Samuelson, P. A. (1958), "An exact consumption-loan model of interest with or without the social contrivance of money," Journal of Political Economy, 66, 467-482.

26. Shapley, L. S. (1976), "Non-cooperative general exchange," in S. A. Y. Lin (ed.), Theory and Measurement of Economic Externalities, Academic Press, 155-175.

27. Shapley, L. S. and M. Shubik (1977), "Trade using one commodity as a means of payment," Journal of Political Economy, 85, 937-968. 